University of Nebraska - Lincoln

DigitalCommons@University of Nebraska - Lincoln

6-2007

\title{
Defect-mediated properties of magnetic tunnel junctions
}

\author{
Julian P. Velev \\ University of Nebraska-Lincoln, julian.velev@unl.edu \\ Mikhail Ye Zhuravlev \\ University of Nebraska-Lincoln, myezhur@gmail.com \\ Kirill D. Belashchenko \\ University of Nebraska-Lincoln, belashchenko@unl.edu \\ Sitaram S. Jaswal \\ University of Nebraska-Lincoln, sjaswal1@unl.edu \\ Evgeny Y. Tsymbal \\ University of Nebraska at Lincoln, tsymbal@unl.edu
}

See next page for additional authors

Follow this and additional works at: https://digitalcommons.unl.edu/physicstsymbal

Part of the Condensed Matter Physics Commons

Velev, Julian P.; Zhuravlev, Mikhail Ye; Belashchenko, Kirill D.; Jaswal, Sitaram S.; Tsymbal, Evgeny Y.; Katayama, T.; and Yuasa, S., "Defect-mediated properties of magnetic tunnel junctions" (2007). Evgeny Tsymbal Publications. 9.

https://digitalcommons.unl.edu/physicstsymbal/9

This Article is brought to you for free and open access by the Research Papers in Physics and Astronomy at DigitalCommons@University of Nebraska - Lincoln. It has been accepted for inclusion in Evgeny Tsymbal Publications by an authorized administrator of DigitalCommons@University of Nebraska - Lincoln. 


\section{Authors}

Julian P. Velev, Mikhail Ye Zhuravlev, Kirill D. Belashchenko, Sitaram S. Jaswal, Evgeny Y. Tsymbal, T. Katayama, and S. Yuasa 


\title{
Defect-Mediated Properties of Magnetic Tunnel Junctions
}

\author{
J. P. Velev ${ }^{1}$, M. Ye. Zhuravlev ${ }^{1}$, K. D. Belashchenko ${ }^{1}$, S. S. Jaswal ${ }^{1}$, E. Y. Tsymbal ${ }^{1}$, T. Katayama ${ }^{2}$, and S. Yuasa ${ }^{2}$ \\ ${ }^{1}$ Department of Physics, Center for Materials and Nanoscience, University of Nebraska, Lincoln, NE 68588-0111 USA \\ ${ }^{2}$ National Institute of Advanced Industrial Science and Technology (AIST), Tsukuba, Ibaraki 305-8568, Japan
}

\begin{abstract}
Defects play an important role in the properties of metal oxides which are currently used as barrier layers in magnetic tunnel junctions (MTJs). We study the effect of $O$ vacancies on the interlayer exchange coupling (IEC) and tunneling magnetoresistance (TMR) in Fe-MgO-Fe tunnel junctions. Measurements of IEC in fully epitaxial Fe-MgO-Fe(001) tunnel junctions show IEC is antiferromagnetic (AFM) for small MgO thickness but changes sign and then vanishes for large barrier thickness. First-principles calculations based on density functional theory demonstrate that the presence of neutral $O$ vacancies $(F$-centers) in the MgO barrier can explain this behavior. Resonant tunneling through the $F$-centers makes IEC AFM for thin barriers but with increasing MgO thickness the resonance contribution to IEC is reduced resulting in the ferromagnetic (FM) coupling typical for perfect $\mathrm{MgO}$ barriers. First-principles calculations also show that $\mathrm{O}$ vacancies can affect TMR. $F$-centers produce occupied localized $s$-states and unoccupied resonant $p$-states in the gap of MgO. We demonstrate that $F$-centers affect the conductance by either resonant transmission or nonresonant scattering of tunneling electrons both causing a substantial reduction of TMR compared to the ideal case.
\end{abstract}

Index Terms-Heterostructures, magnetic coupling, magnetic memories, magnetic recording.

\section{INTRODUCTION}

$\mathbf{S}_{\mathrm{p}}^{\mathrm{p}}$ PIN-BASED electronics has a potential for exciting new applications [1]. Magnetic tunnel junctions (MTJs) look promising for magnetic recording and computer memory applications. An MTJ consists of two ferromagnetic (FM) electrodes magnetically decoupled by a thin insulating barrier [2]. The change of the electrical resistance of the MTJ with a change in the relative magnetization orientation of the electrodes is known as tunneling magnetoresistance (TMR). The interlayer exchange coupling (IEC) is a measure of the magnetic coupling between the two FM layers [3].

Since the first observation of large reproducible TMR at room temperature in amorphous $\mathrm{Al}_{2} \mathrm{O}_{3}$-based MTJs [4], there has been a surge in research in this field. In particular, giant TMR values of more than $1000 \%$ were predicted, theoretically, in MTJs based on crystalline $\mathrm{MgO}$ barriers epitaxially grown on $\mathrm{Fe}(001)$ electrodes [5], [6]. This prediction was followed by experimental realizations of MTJs utilizing both epitaxial and textured $\mathrm{Fe}$ and $\mathrm{CoFe}$ electrodes [7], [8] in which more modest TMR values of up to $300 \%$ were obtained at low temperatures. Several sources for this discrepancy mainly related to interface imperfections have been investigated such as oxidation [9] and roughness [10]. Although the interface plays an important role, defects in the insulator can also influence the properties of MTJs [11], [38]. In particular, it was found that impurity-assisted resonant tunneling can reverse TMR [12], [13].

IEC was discovered in metallic multilayers [14] and was found to oscillate as a function of spacer thickness [15]. Experimental observations of IEC across an insulator are still rare [17], [16]. The theoretical approaches to IEC are based either on the spin torque exerted by one ferromagnet on the other [18], [19] or the induced DOS in the spacer by the ferromagnets [20], [21]. For a metallic spacer, the theory predicts an oscillatory

Digital Object Identifier 10.1109/TMAG.2007.893311

Color versions of one or more of the figures in this paper are available online at http://ieeexplore.ieee.org. coupling and relates the period of the oscillations to the spanning vectors of the Fermi surface of the spacer material. For an insulating spacer, a nonoscillatory dependence is expected with the strength of IEC exponentially decreasing as a function of barrier thickness. The experimental results significantly differ from the theoretical predictions for ideal MTJs both in the magnitude and the sign of IEC. The presence of impurities or defects in the barrier may significantly influence IEC [22], [23].

Defects in metal oxides have been exhaustively studied in quantum chemistry because they determine the chemical properties of the oxides [24]. Although defects must play a crucial role in real MTJs with oxide barriers, first-principles models so far have mostly been limited to ideal MTJs. In this paper, we use large scale first-principles calculations based on density functional theory to elucidate the effect of neutral $\mathrm{O}$ vacancies ( $F$-centers) on IEC and TMR in crystalline $\mathrm{Fe}-\mathrm{MgO}-\mathrm{Fe}(001)$ MTJs. We find that resonant tunneling through the $F$-centers makes IEC AFM for thin barriers. This explains the observed IEC as a function of $\mathrm{MgO}$ barrier thickness in fully epitaxial $\mathrm{Fe}-\mathrm{MgO}-\mathrm{Fe}(001)$ MTJs. We also show that $\mathrm{O}$ vacancies affect the conductance by either resonant transmission or nonresonant scattering of electrons both causing a substantial reduction of TMR.

\section{VACANCIES IN MGO}

It has been well established that the properties of metal oxides are strongly influenced by the presence of defects [24]. O vacancies are of special interest because they are common to all oxides. Bulk $F$-centers were studied extensively using optical spectroscopy [25]. Recently, $F$-centers in thin $\mathrm{MgO}$ films were investigated using electron paramagnetic resonance [26] and scanning tunneling spectroscopy [27]. There is continuing theoretical interest in the physical properties of $F$-centers in $\mathrm{MgO}$ in the bulk [28], [29] and on the surface [30].

We use a pseudopotential plane-wave method (VASP) [31] within the local density approximation (LDA) to study the electronic structure of $F$-centers in the bulk $\mathrm{MgO}$ and in the barrier layer of $\mathrm{Fe}-\mathrm{MgO}-\mathrm{Fe}$ MTJs. In bulk $\mathrm{MgO}$, vacancies are introduced by removing a neutral $\mathrm{O}$ atom in a 32, 64, and 216 

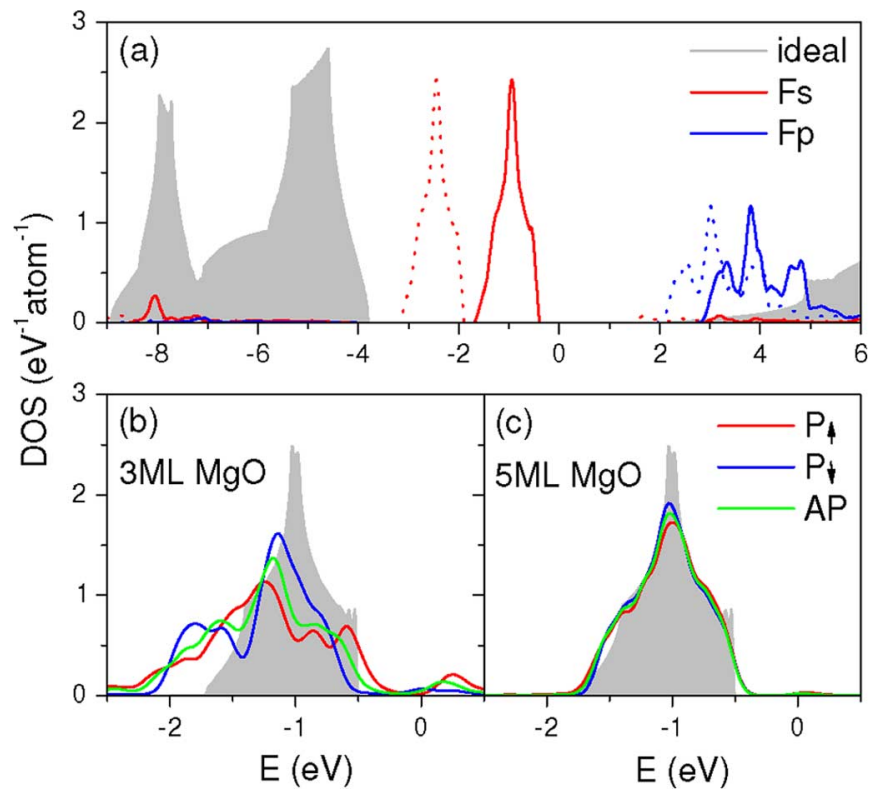

Fig. 1. DOS of (a) bulk $\mathrm{MgO}$ (shaded area) and $F$-center $s$ and $p$-states for a (1) $/(32)$ cell (solid line). The dashed line indicates the approximate position of the levels corrected for self-interaction; (b) and (c) $F$-center $s$-state in bulk $\mathrm{MgO}$ (shaded area) and $\mathrm{Fe}-\mathrm{MgO}-\mathrm{Fe}$ MTJ (majority $\left(\mathrm{P}_{\uparrow}\right)$ and minority $\left(\mathrm{P}_{\downarrow}\right)$ in parallel and antiparallel (AP) orientation of the magnetization in the electrodes). Both cells have $(1 / 4)$ vacancy concentration in plane. $E=0$ is the position of the Fermi level in Fe-MgO-Fe MTJs.

atom supercells. ${ }^{1}$ Atomic relaxations show that the $\mathrm{MgO}$ lattice is only slightly distorted in the vicinity of the vacancy. Fig. 1(a) shows the calculated density of states (DOS) of an $F$-center superimposed on DOS of bulk $\mathrm{MgO}$. The $\mathrm{O}$ vacancy produces two localized states, one above and one below the Fermi level $\left(E_{F}\right)$. The first state has $s$ character and it is occupied by two electrons on the $F$-center site. The $s$ character is evident from the partial DOS as well as from the spherically symmetric charge density on the vacancy site seen in Fig. 2(a). The charge density plot reveals significant hybridization only between the vacancy $s$-state and $p$-states of the nearest $\mathrm{O}$ ions. The second state is an unoccupied $p$-state which appears on the $F$-center site at the bottom of the $\mathrm{MgO}$ conduction band (Fig. 1 ). Since the $F$-center $p$-state lies within the continuum of the conduction-band states, a nonzero charge density is present on atoms far away from the vacancy site [Fig. 2(b)]. Our results are consistent with the earlier calculations of the electronic structure of $\mathrm{O}$ vacancies in $\mathrm{MgO}$ [28].

These results conform with the experimental data [26], [27] where two defect levels are observed in the band gap of $\mathrm{MgO}$ [26, Fig. 3(a)], [27, Fig. 3(c)]—one centered a few electronvolts above the valence band maximum (VBM) and another overlapping with the conduction band minimum. The first level can be identified with the $F$-center ground $s$-state while the second corresponds to the $F$-center exited $p$-state. It is unlikely that the occupied localized level represents a $V$-center formed by a neutral $\mathrm{Mg}$ vacancy, as suggested in [27], because the formation energy of a $V$-center is substantially larger than that of an $F$-center

${ }^{1}$ Supercells are tetragonal for the 32 atom cell $2 a_{\mathrm{Fe}}(1,1, \sqrt{2})$ and cubic for the 64 and 216 atom cells with edge size $2 a_{\mathrm{MgO}}$ and $3 a_{\mathrm{MgO}}$, respectively. $a_{\mathrm{Fe}}=2.87 \AA$ is the experimentally observed lattice constant of $\mathrm{Fe}$ and $a_{\mathrm{MgO}}=\sqrt{2} a_{\mathrm{Fe}}$.
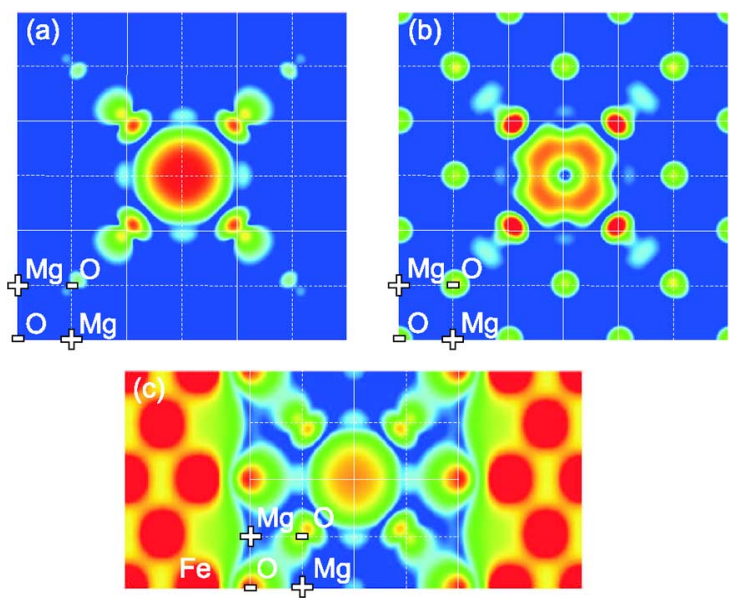

Fig. 2. Charge density calculated in the energy window containing the defect level (a) and (b) $\mathrm{MgO} s$ - and $p$-states ((1)/(216) cell); (c) Fe-MgO-Fe(001) with five layers of $\mathrm{MgO}$ and one $\mathrm{O}$ vacancy in the middle $\mathrm{MgO}$ layer $((1) /(8)$ cell). Vacancy is in the middle, the positions of the $\mathrm{Mg}(+)$ and $\mathrm{O}(-)$ atoms are indicated for one unit cell. Exponential scale is used with red indicating high and blue low charge density (arbitrary units).

[30]. The exact position of the occupied level varies in experiments depending on the exact environment of the vacancy and the size of the gap which is smaller close to the surface. Theoretically, the distance of the occupied level from the VBM can be understood as an upper limit. For highly localized electrons on the $F$-center, self-interaction corrections become important. The self-interaction energy has been estimated to be $U_{s s}=1$ to $1.5 \mathrm{eV}$ and $U_{s p}=0.5$ to $0.8 \mathrm{eV}$ [28]. Fig. 1(a) shows the approximate positions of the $F$-center levels after the self-interaction has been subtracted which agree well with the experimental data.

To simulate vacancies in the barrier layer of $\mathrm{Fe}-\mathrm{MgO}-\mathrm{Fe}$ MTJs, we consider a supercell with one vacancy per 4 or $8 \mathrm{O}$ atoms in the plane in the middle layer of the junction. The occupied vacancy level appears centered around $-1 \mathrm{eV}$. Its width depends strongly on the vacancy density and the interaction with the electrodes. Fig. 1(b) and (c) shows $F$-center levels in $\mathrm{Fe}-\mathrm{MgO}-\mathrm{Fe}$ MTJs with 3 and 5 atomic layers (MLs) of $\mathrm{MgO}$, respectively, compared to the $F$-center in bulk $\mathrm{MgO}$ with comparable vacancy density. The broadening of the level is intrinsic (from interaction with the other vacancies) and extrinsic (from interaction with the Fe electrodes). Fig. 1(c) demonstrates that if there is more than 2 MLs between the vacancy and the electrode, the interaction with the electrodes is weak and the broadening is purely intrinsic. If the vacancy is closer, the level is broadened due to large escape rates to the electrodes and the broadening is spin-dependent Fig. 1(b). In Fig. 2(c), the charge density in an $\mathrm{Fe}-\mathrm{MgO}-\mathrm{Fe}$ MTJs with five MLs of $\mathrm{MgO}$ is shown. The vacancy interacts only with the $\mathrm{O}$ on the neighboring layers and does not affect the $\mathrm{O}$ atoms on the surface.

\section{IEC}

The IEC energy per unit area is given by $E_{\mathrm{IEC}}=-J \cos \theta$, where $\theta$ is the angle between the magnetizations of the two FM layers. Theoretical models have been quite successful in explaining IEC observed in metallic spacers [3]. Unlike a metallic spacer layer, the tunneling barrier leads to nonoscillatory coupling which decays exponentially as a function of the barrier 


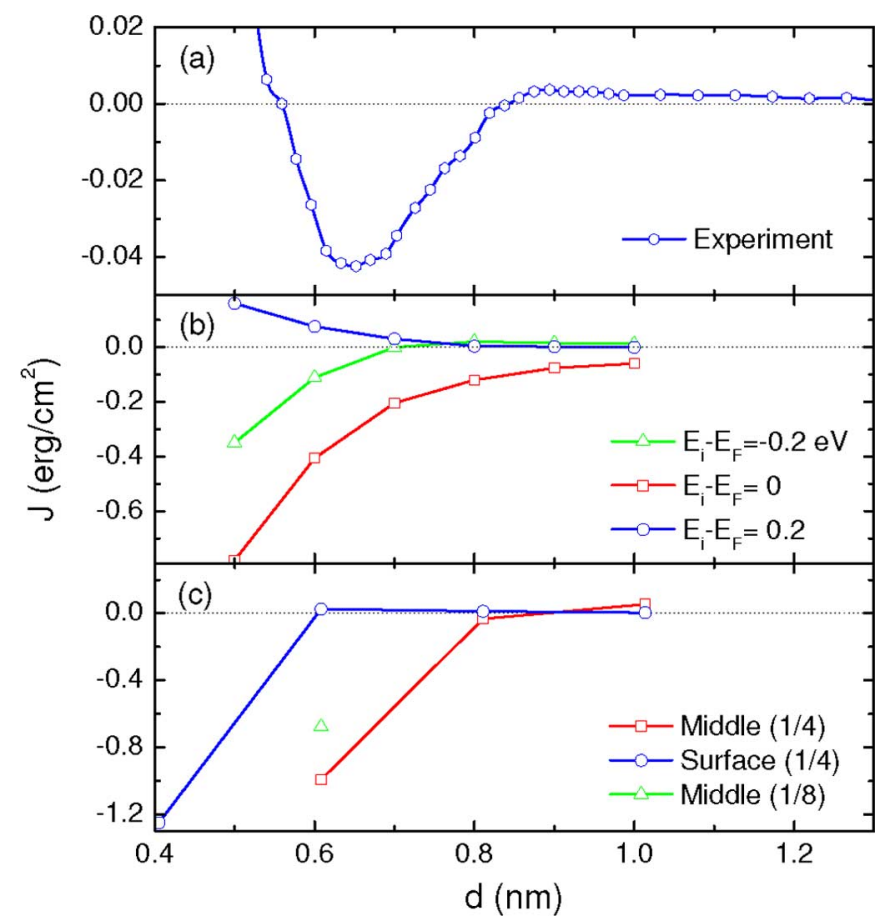

Fig. 3. IEC in $\mathrm{Fe}-\mathrm{MgO}-\mathrm{Fe}(001)$ junctions versus $\mathrm{MgO}$ thickness (a) measured with 15-nm free Fe layer, (b) calculated from the free electron model and averaged over the impurity position, and (c) calculated ab initio in VASP for a single $\mathrm{O}$ vacancy.

thickness [18], [19], [20], [21]. Experimental observations of IEC have been reported for only two systems- $\mathrm{Fe}-\mathrm{MgO}-\mathrm{Fe}$ [16] and $\mathrm{Fe}-\mathrm{Si}-\mathrm{Fe}$ [17]. In both cases large coupling strength is observed comparable to that of metallic spacers. Also, a model for a defect-free $\mathrm{Fe}-\mathrm{MgO}-\mathrm{Fe}$ predicts IEC to be FM at thickness less than $10 \AA$ [22], [23]. Thus, experimental results differ from theoretical prediction both in magnitude and sign of the coupling.

\section{A. Experiment}

IEC in Fe-MgO-Fe MTJs was found to be AFM for small barrier thickness but changes sign for thicker barriers [16]. It is not clear, however, whether the switching is caused by magnetostatic interaction due to interface roughness or has intrinsic origin. In order to clarify this, we prepared fully epitaxial $\mathrm{Fe}-\mathrm{MgO}-\mathrm{Fe}(001)$ MTJs with wedge-shaped $\mathrm{MgO}$ by molecular beam epitaxy (MBE). The flatness of the interfaces was controlled by observing in situ the reflective high-energy electron diffraction (RHEED) pattern. Longitudinal magneto-optical Kerr effect (MOKE) was used to measure hysteresis loops of the free and pinned Fe layers at room temperature. Further details on synthesis and characterization of the MTJs can be found in [32].

Fig. 3(a) shows the measured dependence of IEC on $\mathrm{MgO}$ thickness. The coupling is large FM for $\mathrm{MgO}$ thickness below $0.3 \mathrm{~nm}$ due to pinholes. It becomes AFM for $\mathrm{MgO}$ thickness range of about $0.5-0.8 \mathrm{~nm}$; then, it reverses to weak FM above $0.8 \mathrm{~nm}$. For thicker barriers, IEC approaches gradually to zero. We find that the absolute value of IEC increases with increasing Fe free layer thickness $(15,20$, and $30 \mathrm{~nm})$. This indicates that the better quality and interface flatness of the $\mathrm{MgO}$ wedge, achieved by increasing the thickness of the bottom Fe layer, may provide a stronger AFM coupling.

It should be pointed out that in [16] the coupling for larger barrier thickness approaches a finite value. This is attributed to the "orange peel" effect which given the quality of the sample is nearly constant at this thickness range. In our case, the coupling approaches zero in the same range of thicknesses (above $1.5 \mathrm{~nm}$ ). Therefore, the magnetostatic interaction due to interface roughness is negligible as a consequence of the high-quality $\mathrm{Fe}-\mathrm{MgO}$ interfaces and the small area of the sample at a given thickness. This excludes the "orange peel" coupling effect as the origin of the sign change of IEC.

\section{B. Model}

The change of the sign of IEC indicates that there is an AFM coupling mechanism which competes at small thickness with the FM coupling expected for ideal barriers. This can be explained by localized defect states in the barrier.

In order to demonstrate that, we calculate IEC between two semifinite FM layers separated by a plane barrier layer of thickness $d$ from the spin current using the spin torque formalism [18], . The electronic structure of the ferromagnets is modeled by free-electron bands exchange-split by $\Delta_{\mathrm{ex}}$. The barrier is represented by a rectangular potential of height $U_{b}$ in which a $\delta$ function located in the barrier at position $r_{i}$ represents the defect. This potential creates a quantum well containing an impurity level of energy $E_{i}$ [22], [23].

Fig. 3(b) shows IEC as a function of the barrier thickness. The coupling can be FM or AFM depending on the position of the defect level. If the level is below $E_{F}$, the coupling is AFM but its magnitude is larger when the level is closer to the $E_{F}$. The absolute values of IEC are significantly higher than those in the same system without impurities and proportional to the defect concentration.

The origin of the AFM exchange coupling can be understood from energy-resolved contributions to the spin current from majority and minority spin electrons shown in Fig. 4(a) and (b). The spin current has a pronounced resonant character which correlates with the local DOS shown in Fig. 4(c) and (d). The width of the impurity levels is determined by the density of metal-induced gap states at the defect position, which is larger for majority spins. The net spin current is integrated over all energies up to $E_{F}$ and summed over the two spin contributions. If the defect level is filled (lies well below $E_{F}$ ) the net spin current appears to be majority-dominated resulting in an FM exchange coupling. However, when the defect level is partially filled (lies near $E_{F}$ ) the minority spin current exceeds the majority spin current due to the incomplete contribution from the resonant peaks of different width leading to an AFM coupling.

\section{Ab Initio}

It follows from our model considerations that resonant tunneling through partially filled defect levels can mediate AFM coupling. In order to confirm that this is the mechanism of the observed variation of IEC as a function of $\mathrm{MgO}$ thickness, we perform $a b$ initio calculations of the coupling for ideal $\mathrm{Fe}-\mathrm{MgO}-\mathrm{Fe}$ MTJs and for junctions which contain $\mathrm{O}$ vacancies [32].

IEC is a difference in the total energy between parallel $(\mathrm{P})$ and antiparallel (AP) magnetization of an MTJ, $J=\left(E_{\mathrm{AP}}-E_{\mathrm{P}}\right) / 2$. 


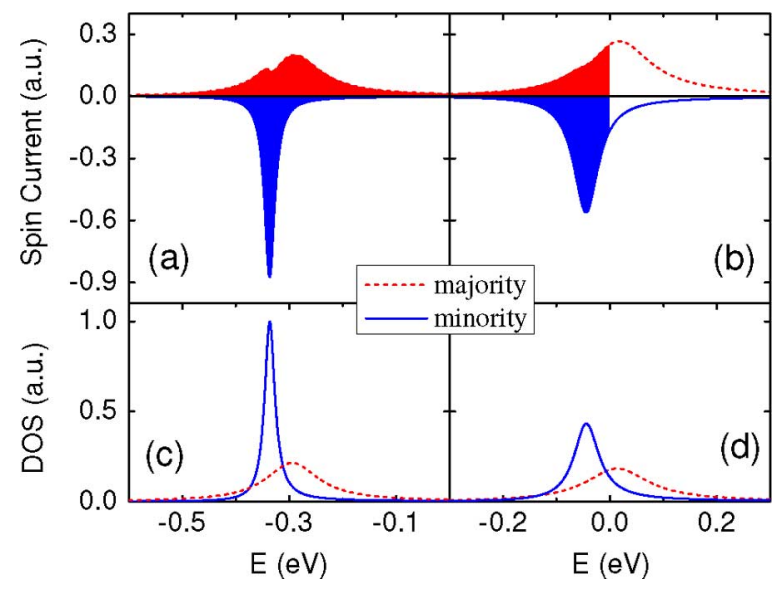

Fig. 4. Energy-resolved contributions to the spin current from majority and minority spin electrons incident from (a) and (b) the left ferromagnet and (c) and (d) DOS at the impurity position $r_{i}$ for (a) and (b) $E_{i}=-0.3 \mathrm{eV}$ and (c) and (d) $E_{i}=0 \mathrm{eV}$. Shadow regions show filled states which contribute to the spin current at $T=0, d=8 \AA$, and $r_{i}=3 \AA$.

We use VASP to calculate the total energy in a double supercell. ${ }^{2}$ In agreement with the model predictions for an ideal $\mathrm{MgO}$ barrier, we find that IEC is FM [Fig. 3(c)] and decays almost perfectly exponentially with $\mathrm{MgO}$ thickness [32]. From the slope of the graph, we find that the decay rate of IEC is $0.4 \AA^{-1}$. This value agrees very well with the slope of the conductance against $\mathrm{MgO}$ thickness [33] and coincides with the decay rate of the $\Delta_{1}$-state in the complex band structure of $\mathrm{MgO}$ [34]. This indicates that the main contribution to IEC comes from the electrons around the Fermi surface. This behavior is in agreement with theoretical predictions [18], [19], [20], [21].

Then, we calculate IEC in the presence of $\mathrm{O}$ vacancies assuming that they lie within one monolayer of $\mathrm{MgO}$, either in the middle or on the surface of the $\mathrm{MgO}$ slab. It is seen from Fig. 3(c) that the coupling is AFM for small MgO thickness but changes sign at larger thickness. IEC is stronger when the vacancy lies in the middle and increases with vacancy concentration. At large thickness, the coupling becomes comparable to that of the ideal system. Thus, the presence of the vacancy provides the AFM coupling which wins at small thicknesses. Moreover, the calculated DOS on the vacancy shown in Fig. 1(b) and (c) indicates that the mechanism of the AFM coupling is indeed resonant tunneling through the $F$-center. For three MLs of $\mathrm{MgO}$ [Fig. 1(b)], the vacancy levels are broadened by the interaction with the electrodes and partially filled because their width becomes larger than the distance to the Fermi level. The broadening on the majority-spin state is larger than that of the minority-spin state due to much larger escape rates through the majority $\Delta_{1}$-state. This provides the basis for spin current imbalance and AFM coupling. For five MLs of $\mathrm{MgO}$, the vacancy only weakly interacts with the leads [Fig. 1(c)] which returns the coupling to FM. The short range of the resonant tunneling through the $F$-center explains why the AFM coupling wins only for very small thickness.

\footnotetext{
${ }^{2}$ Supercells are tetragonal $\mathrm{Fe}_{5}-\mathrm{MgO}_{n}-\mathrm{Fe}_{5}-\mathrm{MgO}_{n}$. The $(1 / 8)$ cell sizes are $\left(2 a_{\mathrm{MgO}}, 2 a_{\mathrm{MgO}}, 5 a_{\mathrm{Fe}}+(n / 2) a_{\mathrm{MgO}}+2 h\right)$, where $h=2.17 \AA$ is the distance between $\mathrm{Fe}$ and $\mathrm{O}$ atoms at the interface [5].
}

\section{TMR}

TMR is defined as the normalized change of the electrical resistance of the MTJ with a change in the relative magnetization orientation of the electrodes, TMR $=\left(G_{\mathrm{P}}-G_{\mathrm{AP}}\right) / G_{\mathrm{AP}}$. Theoretically, TMR values greater than $1000 \%$ are predicted for not too small $\mathrm{MgO}$ thickness [5], [6], [33]. This is explained by the dominant contribution to the conductance of the $\Delta_{1}$ band in Fe which is spherically symmetric in the plane of the layers and matches well with the lowest $\mathrm{MgO}$ conductance band ( $\mathrm{Mg} s$-like) resulting in low rate of decay in the insulator [5]. The other states have to tunnel through higher lying bands resulting in higher barrier heights and much higher decay rates. The asymmetry in the energy interval $(-1,1.5) \mathrm{eV}$ where $\Delta_{1}$ appears in only in the majority spin channel, results in $G_{\mathrm{P}} \gg G_{\mathrm{AP}}$ and giant TMR.

\section{A. Model}

From inspection of the barrier DOS with defects [Fig. 1(a)], it is immediately obvious that there are two regions in which the transport properties can be expected to be essentially different. In the energy range close to the defect levels, the conductance is dominated by resonant tunneling. The resonant conductance in each spin channel is given by [13], [35]

$$
G_{\sigma} \propto \frac{\Gamma_{L \sigma} \Gamma_{R \sigma}}{\left(E-E_{d}\right)^{2}+\left(\Gamma_{L \sigma}+\Gamma_{R \sigma}\right)^{2} / 4}
$$

where $\Gamma_{L \sigma}$ and $\Gamma_{R \sigma}$ are the electron escape rates to the left and right electrodes and $E_{d}$ is the position of the defect level. The escape rates is $\Gamma_{\sigma}=\rho_{\sigma} e^{-2 \kappa x}$ where $x$ is defect position and $\rho_{\sigma}$ DOS in the electrode [13]. Taking into account that the electrodes on either side are the same and the vacancy is in the middle, $\Gamma_{L \sigma}=\Gamma_{R \sigma} \propto \rho_{\sigma}$.

Then, at resonance $\left(E=E_{d}\right), \mathrm{TMR}=\left(\rho_{\uparrow}-\rho_{\downarrow}\right)^{2} / 4 \rho_{\uparrow} \rho_{\downarrow}$. Thus, the TMR ratio is proportional to the spin asymmetry (including all states, not just the $\Delta_{1}$ ) in the electrodes which is typically not very large. This is applicable to $O$ vacancies lying not too far from the electrodes so that all the transverse states contribute to the conductance. Thus, the resonant mechanism of tunneling leads to moderate values of TMR.

Off resonance tunneling is determined by the lowest decay rate in the insulator. The decay rate, $\kappa=\sqrt{2 m^{*}(U-E) / \hbar^{2}}$, is determined by the barrier height and the effective mass. The barrier height is not affected by the removal of an $\mathrm{O}$ atom but the effective mass is inversely proportional to the hopping integral $t \propto \hbar^{2} /\left(2 m^{*} a^{2}\right)$ where $a$ is the lattice constant. The absence of an $\mathrm{O}$ atom lowers hopping through that site and overall increases the effective mass and the decay rate.

\section{B. Ab Initio}

Model considerations suggest that TMR is reduced in the presence of defects in the barrier. We calculate the tunneling conductance in $\mathrm{Fe}-\mathrm{MgO}-\mathrm{Fe}(001)$ MTJs with $\mathrm{O}$ vacancies using self-consistent potentials obtained within the tight-binding linear muffin-tin orbital (TB-LMTO) method in the atomic sphere approximation (ASA) [36]. To represent an O vacancy, we replace an $\mathrm{O}$ atom by an empty sphere of equal radius. The TB-LMTO electronic structure is compared to VASP calculations for all systems showing excellent agreement. Then, the two-terminal conductance is obtained using the principal-layer Green function technique [33], [37]. 


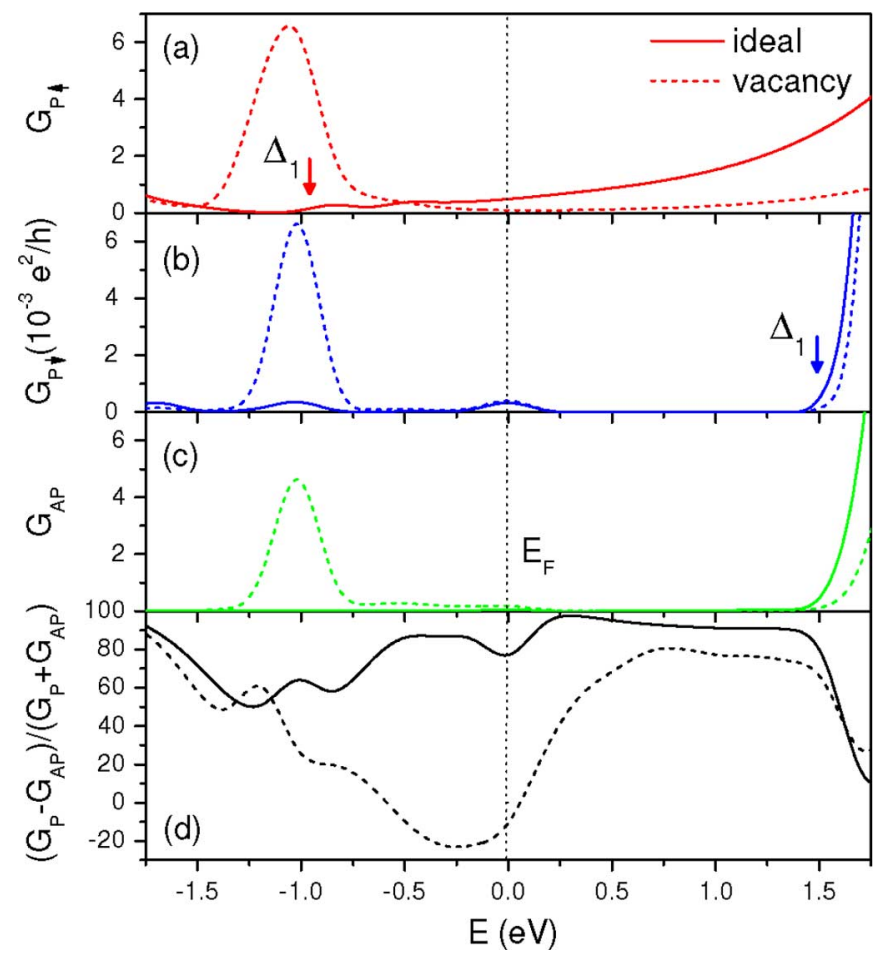

Fig. 5. Conductance per cell and TMR in $\mathrm{Fe}-\mathrm{MgO}-\mathrm{Fe}(001)$ with five layers of $\mathrm{MgO}$ for ideal $\mathrm{MgO}$ and $\mathrm{MgO}$ with $\mathrm{O}$ vacancies $((1 / 8)$ cell). (a) Majority-spin and (b) minority-spin in parallel, and (c) either spin in antiparallel configuration conductance. (d) TMR (bound). Arrows indicate the bottom of the majority and minority $\Delta_{1}$ bands.

Fig. 5(a)-(c) shows conductance as a function of energy for an ideal Fe-MgO-Fe MTJ (solid lines) and for an MTJ with O vacancies (dashed lines). For the ideal MTJ, the conductance plots reveal two characteristic energy points: $E \approx-1 \mathrm{eV}$ for majority-spin electrons and $E \approx 1.5 \mathrm{eV}$ for minority-spin electrons [indicated by arrows in Fig. 5(a) and (b)]. Above these energies the conductance increases significantly due to the occurrence of the $\Delta_{1}$ symmetry bands in the electronic structure of Fe. In the energy interval $(-1,1.5) \mathrm{eV}$, the $\Delta_{1}$ band is present only in the majority-spin channel resulting in large values of TMR [5], [6].

$\mathrm{O}$ vacancies produce a pronounced peak in the conductance centered around $E=-1 \mathrm{eV}$ [Fig. 5(a)-(c)]. This peak is due to resonant tunneling of majority- and minority-spin electrons via the $F$-center $s$-state. Another peak (not shown) occurs at $E=3.5 \mathrm{eV}$ due to the $p$ resonant state. The width of the peak depends strongly on the vacancy density and on the coupling to the electrodes determined by $\mathrm{MgO}$ thickness [Fig. 1(b) and (c)]. Notably, TMR [Fig. 5(d)] close to the resonance is reduced because resonant transmission is very similar for all channels, as predicted by the model.

Away from the $F$-center levels, the resonant contribution to the conductance is negligible. Nevertheless, the transmission of the majority-spin electrons is strongly affected by the presence of $\mathrm{O}$ vacancies. This is evident from Fig. 5(a) which shows that at and above $E_{F}$ the conductance of an MTJ with vacancies is reduced by a factor of 5-7 depending on energy, as compared to the conductance of a perfect MTJ. The detrimental effect of $\mathrm{O}$ vacancies on the majority-spin conductance is due to scattering of tunneling electrons between states with different transverse

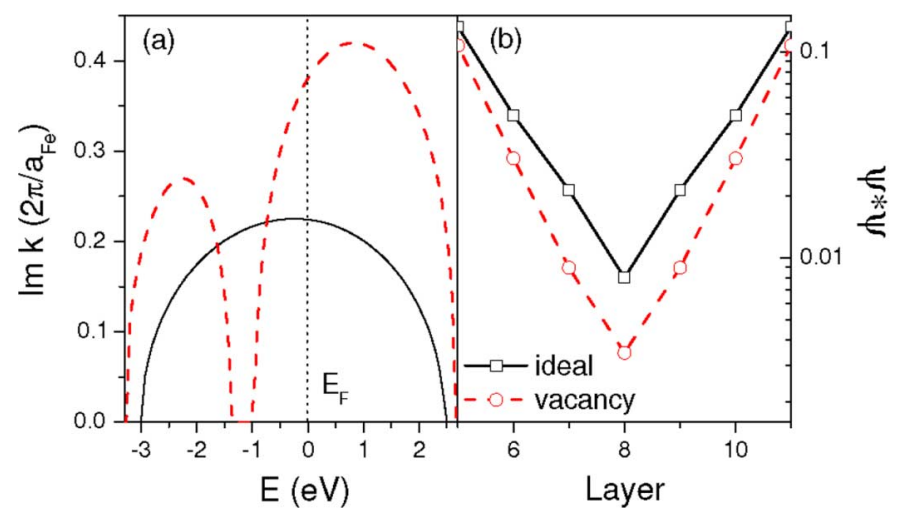

Fig. 6. Decay rate for the $\Delta_{1}$-state in ideal $\mathrm{MgO}$ (solid lines) and $\mathrm{MgO}$ with $\mathrm{O}$ vacancies in (1)/(32) cell (dashed lines) obtained from (a) the complex band structure of bulk $\mathrm{MgO}$ and (b) from the probability density in $\mathrm{Fe}-\mathrm{MgO}-\mathrm{Fe}$. The position of the Fermi energy in $\mathrm{Fe}-\mathrm{MgO}-\mathrm{Fe}$ MTJs is at $E=0 \mathrm{eV}$.

wave vectors $\mathbf{k}_{\|}$. Scattering to states with $\mathbf{k}_{\|} \neq 0$ reduces the transmission coefficient due to a shorter decay length of these states.

The reduction of majority-spin conductance in the presence of $\mathrm{O}$ vacancies can be understood from the complex band structure [34] by analyzing the evanescent $\Delta_{1}$-state which has the lowest decay rate for both ideal and defective $\mathrm{MgO}$ crystals. Fig. 6(a) shows the decay rate of the $\Delta_{1}$ band in an $\mathrm{MgO}$ crystal containing $\mathrm{O}$ vacancies in a periodic arrangement. The vacancy band, which produces the resonant peaks in the conductance, splits the $\mathrm{MgO}$ gap in two parts. Away from resonance, the decay rate of the defective $\mathrm{MgO}$ becomes larger than that of the ideal $\mathrm{MgO}$. This implies that electron scattering from $F$-centers effectively increases the decay constant. A better estimate of the decay rate, accounting for the lack of periodicity in the direction perpendicular to the layers, is to calculate the probability density $|\Psi|^{2}$ for the $\Delta_{1}$-state within the barrier in an Fe-MgO-Fe MTJ [5]. Fig. 6(b) shows $|\Psi|^{2}$ at $E_{F}$. For ideal $\mathrm{MgO}$, the result agrees very well with the complex band structure. The larger slope of the $|\Psi|^{2}$ graph for defective $\mathrm{MgO}$ indicates that the decay rate is about $24 \%$ larger than that for the ideal case. This implies seven times smaller transmission probability which is consistent with the actual results [Fig. 5(a)].

The situation is different for minority-spin electrons because in a perfect $\mathrm{Fe}-\mathrm{MgO}-\mathrm{Fe} \mathrm{MTJ}$ the conductance is not dominated by $\mathbf{k}_{\|}=0$ [33]. In this case, nonresonance scattering by $\mathrm{O}$ vacancies may contribute both destructively and constructively to the conductance. We find that the minority-spin conductance in the parallel configuration of MTJ and the either spin conductance in the antiparallel configuration are small, and $\mathrm{O}$ vacancies do not have a pronounced effect [Fig. 5(b) and (c)].

The overall effect of $\mathrm{O}$ vacancies is the significant reduction of TMR shown in Fig. 5(d) (the bound definition of TMR is used in the graph ${ }^{3}$ ). For ideal junctions, TMR is largely controlled by the enhanced transmission of the $\Delta_{1}$ majority-spin band in the whole energy range $(-1,1.5) \mathrm{eV}$. The conductance through a defective $\mathrm{MgO}$ barrier displays clearly two distinct transport regimes-resonant when the electron energy is close to the position of the vacancy levels and nonresonant when the electron

\footnotetext{
${ }^{3}$ An alternative definition is $\mathrm{TMR}^{\prime}=\left(G_{\mathrm{P}}-G_{\mathrm{AP}}\right) /\left(G_{\mathrm{P}}+G_{\mathrm{AP}}\right)$ which has the advantage of being bound by $\pm 100 \%$. The relationship between the two definitions is $\mathrm{TMR}^{\prime}=\mathrm{TMR} /(2+\mathrm{TMR})$.
} 
energy is far from the vacancy levels. At resonance, the conductance asymmetry arises only from the difference in the majority and minority DOS in the electrodes. Nonresonance scattering from vacancies diminishes the spin asymmetry by reducing the majority conductance.

Quantitative comparison of TMR below $E_{F}$ is hindered by the appearance in the minority channel of interface resonant states [33] which interfere with the tail of the resonant transmission complicating the minority and antiparallel conduction. However, above $E_{F}$, the tendency is very clear and indicative of what is expected in practice. In agreement with previous calculations [5], [6], [33], we find TMR of ideal MTJs $>1800 \%$. TMR in the presence of $\mathrm{O}$ vacancies is reduced to $\lesssim 800 \%$. It is worth mentioning that any defects in the barrier, like Mg vacancies, impurities, dislocations, etc., will act in a similar manner, scattering electrons out of coherence, thus reducing the resultant TMR.

\section{CONCLUSION}

We have found that $\mathrm{O}$ vacancies in the $\mathrm{MgO}$ film affect significantly the properties of $\mathrm{Fe}-\mathrm{MgO}-\mathrm{Fe}$ MTJs. Measurements of IEC in fully epitaxial $\mathrm{Fe}-\mathrm{MgO}-\mathrm{Fe}(001)$ tunnel junctions show IEC is AFM for small $\mathrm{MgO}$ thickness but changes sign and then vanishes for large barrier thickness. Using model and first-principles calculations, we showed that resonant tunneling through $F$-centers makes IEC AFM for thin barriers but with increasing $\mathrm{MgO}$ thickness the resonance contribution to IEC is reduced resulting in the FM coupling typical for perfect $\mathrm{MgO}$ barriers. In addition, first-principles calculations show that $\mathrm{O}$ vacancies can substantially reduce TMR. We demonstrate that $F$-centers affect the conductance by either resonant transmission or nonresonant scattering of tunneling electrons, both causing a substantial reduction of TMR compared to the ideal case. Improving the quality of the $\mathrm{MgO}$ barrier to reduce $\mathrm{O}$ vacancy concentration would improve the properties of $\mathrm{Fe}-\mathrm{MgO}-\mathrm{Fe}$ MTJs.

\section{ACKNOWLEDGMENT}

This work was supported by the National Science Foundation under Grants MRSEC DMR-0213808 and DMR-0203359 and by the Nebraska Research Initiative. The authors would like to thank M. van Schilfgaarde for the use of his TB-LMTO codes. The computations were performed using the Research Computing Facility of the University of Nebraska-Lincoln.

\section{REFERENCES}

[1] S. A. Wolf, D. D. Awschalom, R. A. Buhrman, J. M. Daughton, S. von Molnár, M. L. Roukes, A. Y. Chtchelkanova, and D. M. Treger, Science, vol. 294, pp. 1488-1495, 2001.

[2] E. Y. Tsymbal, O. N. Mryasov, and P. R. LeClair, J. Phys.: Condens. Matter, vol. 15, pp. R109-R142, 2003.

[3] M. Stiles, Ultrathin Magnetic Structures, B. Heinrich and J. A. C. Bland, Eds., III ed. New York: Springer-Verlag, 2005, pp. 99-142.

[4] J. S. Moodera, L. R. Kinder, T. M. Wong, and R. Meservey, Phys. Rev. Lett., vol. 74, pp. 3273-3276, 1995.
[5] W. H. Butler, X.-G. Zhang, T. C. Schulthess, and J. M. MacLaren, Phys. Rev. B, Condens. Matter, vol. 63, 2001, 054416.

[6] J. Mathon and A. Umerski, Phys. Rev. B, Condens. Matter, vol. 63 , 2001, R220 403.

[7] S. S. P. Parkin, C. Kaiser, A. Panchula, P. M. Rice, B. Hughes, M. Samant, and S.-H. Yang, Nature Mater., vol. 3, pp. 862-867, 2004.

[8] S. Yuasa, T. Nagahama, A. Fukushima, Y. Suzuki, and K. Ando, Nature Mater., vol. 3, pp. 868-871, 2004.

[9] X.-G. Zhang, W. H. Butler, and A. Bandyopadhyay, Phys. Rev. B, Condens. Matter, vol. 68, 2003, 092402.

[10] P. X. Xu, V. M. Karpan, K. Xia, M. Zwierzycki, I. Marushchenko, and P. J. Kelly, Phys. Rev. B, Condens. Matter, vol. 73, 2006, 092402.

[11] E. Y. Tsymbal and D. G. Pettifor, Phys. Rev. B, Condens. Matter, vol 58, pp. 432-437, 1998 .

[12] E. Y. Tsymbal and D. G. Pettifor, Phys. Rev. B, Condens. Matter, vol. 64, 2001, 212401.

[13] E. Y. Tsymbal, A. Sokolov, I. F. Sabirianov, and B. Doudin, Phys. Rev Lett., vol. 90, 2003, 186602.

[14] P. A. Grünberg, R. Schreiber, Y. Pang, M. B. Brodsky, and H. Sowers, Phys. Rev. Lett., vol. 57, pp. 2442-2445, 1986.

[15] S. S. P. Parkin, N. More, and K. P. Roche, Phys. Rev. Lett., vol. 64, pp 2304-2307, 1990.

[16] J. Faure-Vincent, C. Tiusan, C. Bellouard, E. Popova, M. Hehn, F. Montaigne, and A. Schuhl, Phys. Rev. Lett., vol. 89, 2002, 107206.

[17] R. R. Gareev, L. L. Pohlmann, S. Stein, D. E. Bürgler, P. A. Grünberg, and M. Siegel, J. Appl. Phys., vol. 93, pp. 8038-8040, 2003.

[18] J. C. Slonczewski, Phys. Rev. B, Condens. Matter, vol. 39, pp. 6995-7002, 1989.

[19] R. Erickson, K. Hathaway, and J. Cullen, Phys. Rev. B, Condens. Matter, vol. 47, pp. 2626-2635, 1993.

[20] P. Bruno, J. Magn. Magn. Mater., vol. 121, pp. 248-252, 1993.

[21] P. Bruno, Phys. Rev. B, Condens. Matter, vol. 52, pp. 411-439, 1995.

[22] M. Ye. Zhuravlev, E. Y. Tsymbal, and A. V. Vedyayev, Phys. Rev. Lett. vol. 94, 2005, 026806.

[23] M. Y. Zhuravlev, J. Velev, A. V. Vedyayev, and E. Y. Tsymbal, J Magn. Magn. Mater., vol. 300, pp. 277-280, 2006.

[24] D. A. King and D. P. Woodruff, Eds., Oxide Surfaces. New York: Elsevier, 2001

[25] Y. Chen, R. T. Williams, and W. A. Sibley, Phys. Rev., vol. 182, pp 960-964, 1969.

[26] M. Sterrer, E. Fischbach, T. Rice, and H. J. Freund, Phys. Rev. Lett., vol. 94, 2005, 186101.

[27] P. G. Mather, J. C. Read, and R. A. Buhrman, Phys. Rev. B, Condens Matter, vol. 73, 2006, 205412.

[28] B. M. Klein, W. E. Pickett, L. L. Boyer, and R. Zeller, Phys. Rev. B, Condens. Matter, vol. 35, pp. 5802-5815, 1987.

[29] A. Gibson, R. Haydock, and J. P. LaFemina, Phys. Rev. B, Condens. Matter, vol. 50, pp. 2582-2592, 1994.

[30] A. M. Ferrari and G. Pacchioni, J. Phys. Chem., vol. 99, pp 17 010-17 018, 1995

[31] G. Kresse and J. Furthmüller, Phys. Rev. B, Condens. Matter, vol. 54 pp. 11 169-11 186, 1996.

[32] T. Katayama, S. Yuasa, J. Velev, M. Y. Zhuravlev, S. S. Jaswal, and E. Y. Tsymbal, Appl. Phys. Lett., vol. 89, p. 112 503, 2006.

[33] K. D. Belashchenko, J. Velev, and E. Y. Tsymbal, Phys. Rev. B, Con dens. Matter, vol. 72, 2005, R140 404.

[34] P. Mavropoulos, N. Papanikolaou, and P. H. Dederichs, Phys. Rev. Lett., vol. 85, pp. 1088-1091, 2000.

[35] S. Datta, Electronic Transport in Mesoscopic Systems. Cambridge, U.K.: Cambridge Univ. Press, 1997.

[36] O. K. Andersen, Phys. Rev. B, Condens. Matter, vol. 12, pp 3060-3083, 1975.

[37] J. P. Velev, K. D. Belashchenko, S. S. Jaswal, and E. Y. Tsymbal, Appl. Phys. Lett., vol. 90, 2007, 072502.

[38] E. Y. Tsymbal and D. G. Pettifor, J. Appl. Phys., vol. 85, p. 5801, 1999.

Manuscript received October 27, 2006; revised February 1, 2007 (e-mail: jvelev@unlserve.unl.edu). 